Journal of Engineering and Applied Sciences 14 (9): 2835-2839, 2019

ISSN: 1816-949X

(C) Medwell Journals, 2019

\title{
A Study on the Effect of Sound for Drowsy Driving Elimination of Highway Tunnel
}

\author{
${ }^{1} \mathrm{Ik}-$ Soo Ahn, ${ }^{1}$ Myung-Jin Bae and ${ }^{2}$ Seong-Geon Bae \\ ${ }^{1}$ Department of Information and Telecommunication, Soongsil University, \\ 369 Sangdo-ro, Dongjak-gu, Seoul, South Korea \\ ${ }^{2}$ School of Software Application, Kangnam University, Gyunggido, Korea
}

\begin{abstract}
Recently as driving accidents on the highway have increased, it has become a social problem. Highway accidents lead to serious accidents due to the rapid speed of the vehicle, resulting in serious injury. Korea Highway Corporation is making a lot of efforts to prevent such highway drowsiness driving accidents. As part of that, the dormant shelters are installed and operated in various places on the outdoor road section. In the tunnel section, various sounds for drowsiness are operated under the name "drowsy alert sound". In particular, the tunnel section is more vulnerable to drowsiness driving because it is a section that must run for a while in a state where the tunnel section is relatively darker than the outdoor section. In this study, we have verified the drowsiness sounds currently used in the highway tunnel section. We used the acoustic method, the brain wave method and the MOS test method for verification. The results will be used as an opportunity to complement and develop the sound used for eliminating drowsiness in existing highway tunnels and to select new sounds.
\end{abstract}

$\underline{\text { Key words: Large accident, sleepy driving, highway tunnel, drowsy alert sound, drowsiness sound, increased }}$

\section{INTRODUCTION}

According to a recent survey, it is said that sleep driving is the highest rate of accidents on highways. In particular, drowsiness driving on highways is not only suicidal but also leads to deaths that can be described as murder. Especially, it is necessary to pay attention to the drowsiness accident occurring in the highway tunnel. There are many tunnels in our country because of the intercultural relationship of $70 \%$ of the country. According to the National Statistical Office (NSO) surveyed in 2015, the number of tunnels across the country is 1,944 and the number of tunnels located on the expressway is about 925. In addition, there are 532 national highways, special metropolitan cities and provinces with 345 tunnels and 133 towns and villages. Among them, highway tunnels are mostly long tunnels compared to other road tunnels. Among them, there is a tunnel designed to reach a length of about $11 \mathrm{~km}$ (10.96 km between Inje and Yangyang). Tunnels are a continuation of boredom that lasts long into an enclosed space of the same design that lasts for quite some time. Such a situation can induce hypnotic effects and further induce drowsiness. In addition, there is no dormitory shelter or shoulder inside the tunnel, so, you have to fight with drowsiness almost unprotected. Driving drowsy in a tunnel causes more severe conditions. According to the statistics of the road traffic corporation surveyed from 2012-2015, the average number of traffic accidents in the tunnel was 640 cases per year. Among them, accidents caused by drowsiness are said to account for the vast majority. To prevent drowsiness in the tunnel, Korea Highway Corporation is currently operating in 254 highway tunnels nationwide under the name of "Drowsy Alert Warning Sound". It provides sound for drowsiness. In this study, the effects of sirens, trumpets and whistles which are the same as the drowsy alert reminders used in Bongpyong tunnel during the highway tunnels, frequency analysis, brain wave analysis and MOS test were performed as a method of veifying drowsiness (Jae-Hoon, 2000; Yoo, 2016; Go and Kim, 1997; Jeong and Bae, 2007; Hong and Bae, 2007; Se-Bin, 2014; Song et al., 2002; Jang et al., 2013).

\section{MATERIALS AND METHODS}

Analysis of sound effects for drowsiness: Sound currently used as a "drowsy reminder alarm" on the highway varies from songs, music, announces, chickens, cuckoos, larks, sirens, trumpets and whistles. Among them, the sound power used in the Bongpyeong tunnel of Yeongdong Expressway is sirens, whistles and trumpets. In this study, we have proved these three sounds through acoustic analysis, EEG analysis and MOS test. In order to

Corresponding Author: Myung-Jin Bae, Department of Information and Telecommunication, Soongsil University, 369 Sangdo-ro, Dongjak-gu, Seoul, Korea 
(a)

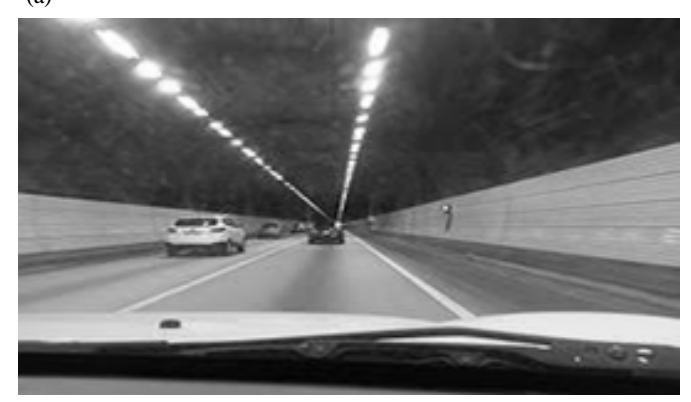

(b)

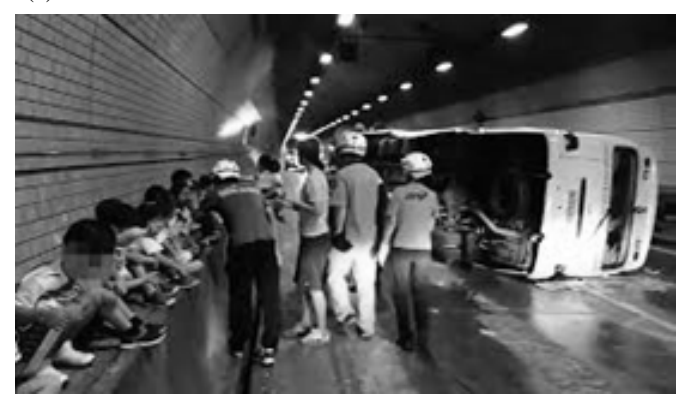

Fig. 1: Drowsy driving accident of highway tunnel: a) highway tunnel and b) Accident of highway tunnel

obtain the accuracy of the analysis of the sound source such sound source was secured and analyzed rather than direct sounding in the internal tunnel of the highway tunnel. First, in the acoustical analysis, fundamental frequency components and energy of the sound were analyzed. We investigated how the sound affects the human body through brain wave analysis. In the Morse test, 5 ordinary people analyzed the response of the sound to the drowsiness sound. Through such analysis and verification, we expect to find a more effective method of eliminating drowsiness (Fig. 1).

Acoustic analysis of sound attenuation: The sound of the siren, the sound of the trumpet and the whistle which are currently being used in the highway tunnel are dynamic and varied in order to wake up the drowsiness. An acoustic analysis was performed to determine what constitutes the sound source. For the acoustic analysis of sound components, we compared and analyzed the spectra of three sounds for drowsiness (Fig. 2 and 3).

Siren sound gradually increases from $400 \mathrm{~Hz}$. It rises strongly at an A point up to the band of $2,000 \mathrm{~Hz}$, gradually descends to a $\mathrm{B}$ and an $\mathrm{C}$ points and appeals widely to a wide bandwidth. The sound of the trumpet rises from $300 \mathrm{~Hz}$ to a large pitch, appealing to the A-C points overall. On the other hand, the whistle sound is very strongly appealing at point $B$, achieving a high pitch with relatively narrow bandwidth only between 2,000 and $3,000 \mathrm{~Hz}$. In addition, all three sounds are strongly stimulated by appealing to various pitches at the C-point which is the high frequency region above $4,000 \mathrm{~Hz}$. Sirens sound stimulates the hearing with relatively low frequency and medium frequency bands. The sound of the trumpet shows a wide range of pitches across the frequency range and stimulates the hearing. The whistle is strong enough to stimulate auditory stimulus in the middle frequency band. All three sounds seem to have acoustical properties that have a stimulating effect on eliminating drowsiness. In the frequency characteristics, the whistle sound shows the most noticeable pitch between 2,000 and $3,000 \mathrm{~Hz}$ which is the most effective audible frequency band and it is judged that the hearing is most certainly stimulated. Secondly, the sirens are wide and high bandwidth from $400 \mathrm{~Hz}-2,000 \mathrm{H}$, stimulating the hearing. Third, the trumpet stimulates the hearing with various pitch projections (Jae-Hoon, 2000; Go and Kim, 1997; Yoo, 2016; Jang et al., 2012; Bum et al., 2012; Moon and Dae, 2015; Kyoung and Jin, 2015).

EEG echo analysis of sleepiness: EEG analysis was performed to verify the sounds of sirens, trumpets and whistles which are used for eliminating drowsiness in highway tunnels. For EEG analysis of the sound for eliminating drowsiness, EEG was measured before listening to the sound. After that, the sirens sound, the trumpet sounds, the whistle sounds and the EEG signals were measured in order. In general, the EEG ratio of people is high in general and the ratio of alpha waves is high. In fact, the EEG peak is peaked in the frequency band corresponding to the Alpha wave.

When the activity of the brain is increased by concentrating on any work or event, EEG waves in the high frequency band such as a high-beta wave are activated. Therefore, we observed changes in alpha, low-beta and high-beta waves according to the sound sources, respectively, based on the mute state. When the sirens were heard on the basis of the EEG in the silent state, the alpha wave decreased by $28.25 \%$, the low-beta wave increased by $41.25 \%$, the high-beta wave increased by $40.28 \%$, the alpha wave decreased by $34.46,7.18 \%$ and high-beta increased by $3.37 \%$. Based on the EEG in the silent state, the alpha wave decreased by $16.83 \%$, the low-beta wave increased by $40.00 \%$, the high-beta wave increased by $52.78 \%$, the alpha wave decreased by 21.77 , $3.59 \%$ and high-beta, $5.62 \%$. When the whistle was heard on the basis of the EEG in the silent state, the alpha wave decreased by $13.55 \%$, the low-beta wave increased by $12.50 \%$, the high-beta wave increased by $19.44 \%$, the 


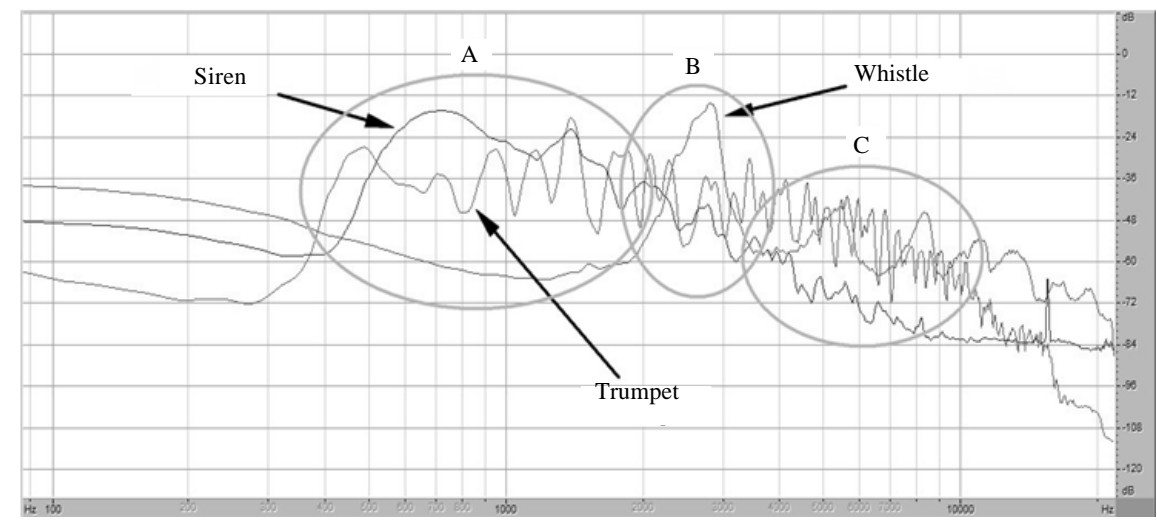

Fig. 2: Spectrum comparative analysis of sound attenuation sound
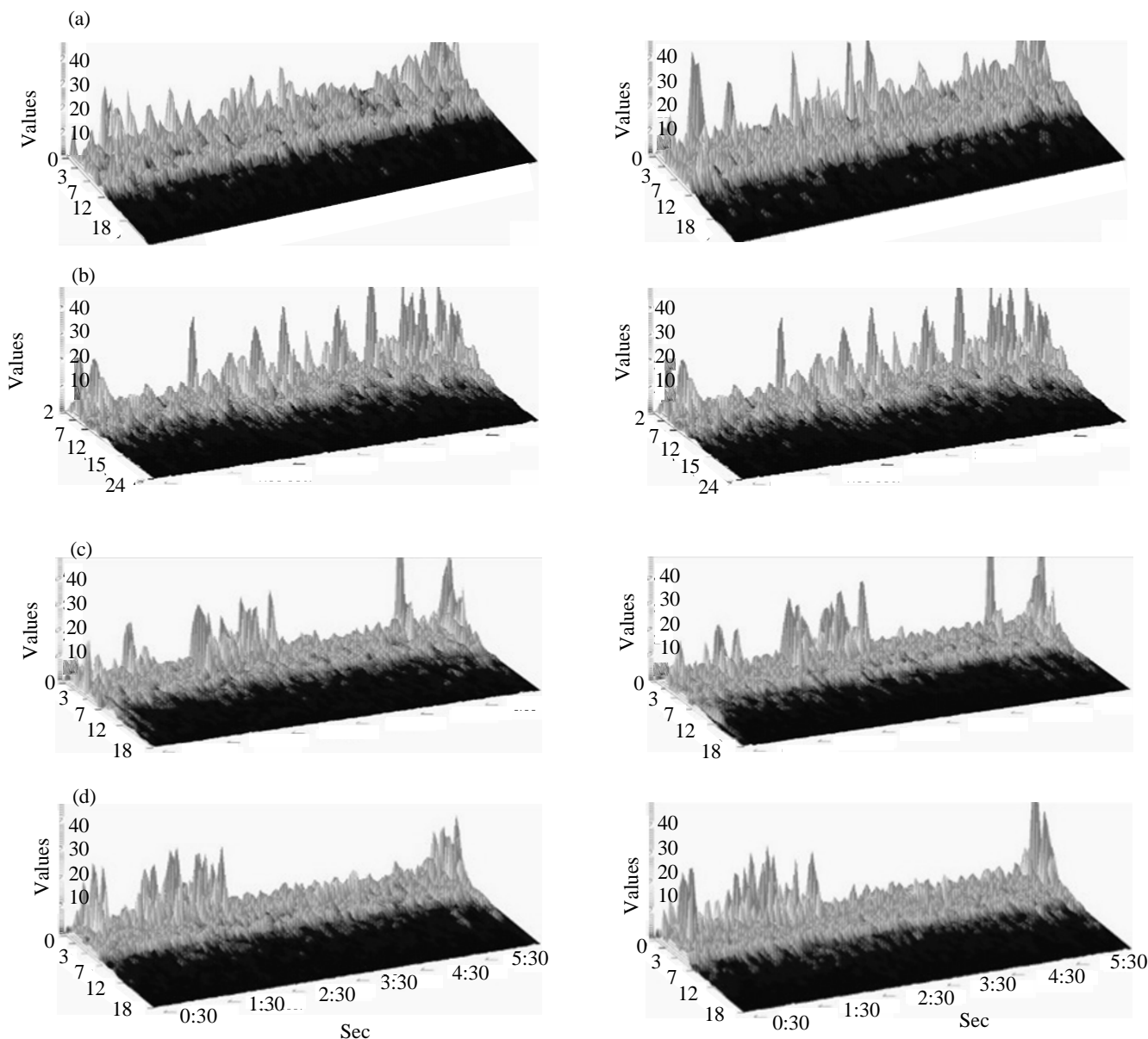

Fig. 3: Comparing EEG analysis before and after hearing of drowsiness sound: a) EEG before hearing a drowsiness; $b$ ) EEG after hearing a siren; c) EEG after hearing a trumpet and d) EEG after hearing a whistle

alpha wave decreased by $16.57,3.08 \%$ and high-beta $2.25 \%$. Experimental results show that when the siren is heard, the reduction of the alpha wave is the largest and the low-beta wave and the high-beta wave are greatly increased. In addition when the whistle was heard, alpha wave decreased the least and low-beta wave and high-beta wave increased the smallest. Therefore, in the EEG measurement when the siren sound is heard, the effect of the drowsiness is the greatest and the effect of the sound of the trumpet and the whistle is decreased. 
Table 1: Morse test result table about three sound for the drowsiness driving inside the expressway

\begin{tabular}{llllll}
\hline Sound absorption for drowsiness & A & B & C & D & E \\
\hline Siren & 4.7 & 4.2 & 4.5 & 4.6 & 4.5 \\
Trumpet & 4.0 & 3.9 & 3.7 & 3.8 & 4.0 \\
Whistle & 3.5 & 3.4 & 3.7 & 4.0 & 3.5 \\
\hline
\end{tabular}

This can be deduced from the fact that the sirens were perceived to be the sounds of police emergencies or emergencies in people which would have stimulated the brain waves most actively. Next, the sound of the trumpet can be inferred from the lightness of the rhythm and the response of the whistle is also perceived by the direction of the police or the state of the state. However, the most weak of all (Go and Kim, 1997; Se-Bin, 2014; Soon-Hee, 2015; Cho and Kim, 2014; Yoo, 2016; Bae and Kim, 2013; Hyung-Jin et al., 2007).

Demonstration through MOS test of sound elimination sleepiness: We heard the sounds of sirens, trumpets and whistles which are sounds for driving drowsy driving in highway tunnels, to 5 drivers and examined their responses. Scoring is a method of assessing the degree to which the sound I hear is effective in eliminating drowsiness and comparing the effect with other sounds. The highest score is 5 points and more than 3 points are judged to be effective. In this way, it is possible to judge what kind of sound is more effective (Table 1).

The listener who listened to the siren sounded the sharpest rhyme and the average score of the MOS test of the five listeners was about 4.5. When the trumpet sound was heard, the average score of the 5 month tests was 3.9 points which was significantly lower than when the sirens were heard. The average score of the whistle was 3.6 which was slightly lower than the trumpet. However, in the case of $\mathrm{C}$ listener in particular, it gave the same score in the sense that the sound of the trumpet and the sound of the whistle were almost equal to the effect of eliminating drowsiness. On the contrary in the case of D listener, unlike other celadons, the whistle sound gave more score in the sense that it would be more effective in eradicating drowsiness than the trumpet sound. Of course, the sirens gave a lot of scores in the sense that the sirens of all five listeners would be most effective in eliminating drowsiness. The results of the EEG analysis are similar to those of the EEG analysis. The siren sound is most intense in unconsciousness and it is considered that the awakening action is greatly shown by strongly recognizing the crisis situation and the warning situation. The sound of the second trumpet is strong, rhythmical and highly concentrated, leading to a great deal of arousal. The whistle sound is likely to be relatively simple and boring. However, in all three sounds, the arousal effect received a high score and was used to be used as a sound for drowsiness. As a result, Morse test verification showed that sirens sound, trumpet sound, whistle sound in the order of EEG results (Yoo, 2016).

\section{RESULTS AND DISCUSSION}

According to the acoustical analysis, it was confirmed that the sound for eliminating drowsiness in the current highway tunnel is composed of the middle frequency and the frequency band in which the human is most likely to perceive in the high frequency range. According to the results of the frequency analysis, the whistle sound has the strongest pitch and it is the sound that stimulates the hearing with a large energy. However, according to EEG analysis and Morse test, the sirens sounded the greatest appeal and showed that they could help the arousal. According to the EEG analysis, the experimenter who listened to the sound for eliminating drowsiness showed that the siren, the trumpet and the whistle were activated in a more awakening manner by activating the alpha wave and beta wave than usual. Also, through the Morse test, most of the subjects responded that they heard the sound for eliminating drowsiness and that they were enough to eliminate drowsiness in the order of sirens, trumpets and whistles. These three analyzes yielded meaningful results and could not help but notice.

The results show that the three kinds of drowsiness sounds differed from the acoustical analysis and showed different results in the electroencephalogram and moss test. From the acoustical point of view, according to frequency analysis, the strongest energy was generated intensively and the sound that stimulated the auditory was the whistle sound but in the EEG and Morse test, we were most strongly imprinted in everyday life and strongly responded to the impressive sound of the siren It is seen. As a result, it was suggested that the sound for eliminating drowsiness should be selected not only by the acoustic analysis but also by choosing or creating a sound in harmony with the emotion. Of course, it was judged that the sound-damping sound that is currently operating in the highway tunnel studied through this study is very effective.

\section{CONCLUSION}

In this study, we analyzed the sound effects used in the tunnel section which is relatively more vulnerable to drowsiness operation. In the results of frequency analysis by acoustic analysis, it was found that the whistle sound among the sounds that rescue drowsiness is strongest in the region where the human auditory frequency band can 
respond most clearly. In the EEG analysis, the sound of eliminating drowsiness stimulated the alpha wave and beta wave and the effect of not only siren sound but also trumpet sound and whistling sound appeared. Morse test, similar to the results of EEG analysis, siren sound, trumpet sound, whistle noise in the order of the possibility of eliminating the drowsy gave a score. These results showed that the sound for eliminating drowsiness harmonically reflects the acoustical contents and the emotional stimulation of human beings, thus, making it more rational. As suggested in the recently announced Highway Tunnel Drowsy Alert Warning Alert Optimization Operation Report, it is thought that people-centered sound, that is pleasant and pleasant sound can be the $m$ ost effective sound for drowsiness. I agree with the suggestion that a warning sound for eliminating drowsiness would be more effective than a coercive, commanding, positive and emotional sound. The results of the study show that all three of the currently active sleeping sound effects are effective for eliminating drowsiness and have the potential to further develop. In this study, the study on the sound for the drowsiness driving of highway tunnels is considered to be a big cornerstone for the research and development of better sound drowsiness in the future. In the future, we will develop and discover sound related to drowsiness and build a more dynamic, dynamic and changeable sound system. In addition, it is intended to develop measures for helping to eliminate drowsiness even when driving on an outdoor road by installing sound inside a car as well as a tunnel by further improving the sound of drowsiness driving.

\section{REFERENCES}

Bae, M. and M. Kim, 2013. Professor Bae's Sound Story. Gimm-Young Publishers, Seoul, South Korean,.

Bum, P., L. Min-Seop and P. Joonhong, 2012. Comparison of sound emission characteristics of diesel engine and gasoline engine sound and artificial engine sound synthesis for sound quality evaluation. Korean Soc. Mech. Eng., 1: 1590-1591.

Cho, G.Y. and Y.H. Kim, 2014. Factors affecting smartphone add iction among university students. J. Korean Acad. Soc. Ind. Sci., 15: 1632-1640.
Go, H.W. and Y.H. Kim, 1997. Setting and analysis of awakening control standard for avoiding sleepy driving. Korean Soc. Auton. Veh. Eng., 2: 1171-1176.

Hong, S.H. and M.J. Bae, 2007. A study on the sound enhancement of concentration. IEICE., 30: 671-672.

Hyung-Jin, P., H. Hyeong-Joo and S. Hyun-Joo, 2007. A study on driver characteristics in a long tunnel using simulator. J. Ergon. Soc. Korea, 26: 89-102.

Jae-Hoon, S., 2000. The validity of legal regulations on the use of mobile phones during driving. Taipei Non-Life Insurance Association, Taipei, Taiwan.

Jang, S.Y., D.J. Park and H.Y. Kohsang, 2012. A study on the effects of smartphone use on driving safety during driving. Korea Transp. Res. Inst., 2012: 75-91.

Jang, S.Y., S.J. Kim and S.S. Goh, 2013. A study on the reduction of traffic accidents by analyzing characteristics of smartphone usage during driving: Applying Ajzen's theory of planned behavior. Kerala State Road Transport Corporation, Thiruvananthapuram, India.

Jeong, C.J. and M.J. Bae, 2007. A study on the classification of amazing sounds. Acoust. Soc. Korea, 26: 57-58.

Kyoung, S.Y. and B.M. Jin, 2015. Psychoacoustic study of automobile engine sound. Acoust. Soc. Korea, 34: 180-180.

Kyu-Young, C. and K. Yoon-hee, 2014. Influencing factors of smartphone addiction in college students. J. Korean Acad. Soc. Ind. Sci., 15: 1632-1640.

Moon, K.B. and Y.Y. Dae, 2015. Design of a green car virtual engine sound generator. Korean Inst. Electr. Eng., 1: 93-94.

Se-Bin, J., 2014. A study on the development of safety system for the elderly driver. J. Korea Soc. Automot. Eng., 22: 234-240.

Song, D.Y., Y.H. Woo and J.S. Kim, 2002. Characteristic analysis of handphone users in driving. Korean Soc. Civ. Eng., 1: 40-44.

Soon-Hee, J., 2015. A study on the relationship between social development and ego resilience of middle school students and smartphone addiction. Master Thesis, Woosuk University, Wanju County, South Korea.

Yoo, H.J., 2016. A study on optimization of sound warning alert for drowsy alert. Intergalactic research Institute for Sound, Korea. 